\title{
CRESCIMENTO, TEORES DE CLOROFILA E DE BIOELEMENTOS EM INDIVÍDUOS JOVENS DE Schinus terebinthifolius EXPOSTOS À POEIRA DE CIMENTO
}

\author{
Wanessa Müller Bujokas ${ }^{1}$, Kelly Geronazzo Martins ${ }^{2}$, Renato Marques ${ }^{3}$

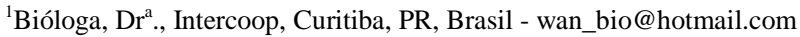 \\ ${ }^{2}$ Bióloga, M.Sc., Doutoranda em Eng. Florestal, UFPR, Curitiba, PR, Brasil - tabebuia77@ hotmail.com \\ ${ }^{3}$ Eng. Florestal, Dr., Depto. Solos e Engenharia Agrícola, UFPR, Curitiba, PR, Brasil - rmarques@ufpr.br
}

Recebido para publicação: 15/05/2009 - Aceito para publicação: 15/10/2009

\begin{abstract}
Resumo
O objetivo deste trabalho foi avaliar o efeito da poeira de cimento no crescimento, nos teores de clorofila e de bioelementos em Schinus terebinthifolius Raddi (Anacardiaceae). Para tanto, dois grupos de plantas com 12 meses de idade foram selecionados, um para controle e o outro para ser submetido à poeira de cimento. Um total de $900 \mathrm{~g}$ de poeira foi pulverizado durante cinco meses, em duas aplicações semanais. Em ambos os grupos de plantas foram realizadas avaliações visuais periódicas para deteç̧ão de possíveis anomalias. Foram avaliados também o crescimento em altura, a área foliar, as massas fresca e seca das folhas, a composição química das folhas e a concentração de clorofila. As plantas pulverizadas apresentaram sintomas de clorose, seguida de necrose nos ápices foliolares e queda das folhas, além de tortuosidade nos caules. Mostraram também aumento significativo nos teores de clorofilas a, b e total, bem como de macro, micronutrientes, $\mathrm{Al}$ e $\mathrm{Na}$, principalmente nas folhas que não passaram pelo processo de lavagem antes da realização das análises. As massas fresca e seca das folhas foram menores nos indivíduos pulverizados. Os resultados indicam que a espécie utilizada apresenta sensibilidade à contaminação com poeira de cimento, podendo ser usada em estudos de biomonitoramento.

Palavras-chave: Aroeira-vermelha; poluição, nutrição florestal; biomonitoramento ativo.
\end{abstract}

\begin{abstract}
Growth, chlorophyll and bio-elements concentration in seedlings of Schinus terebinthifolius exposed to cement dust. The aim of this work was to evaluate the effect of cement dust on the growth, chlorophyll and bio-elements concentrations in Schinus terebinthifolius Raddi (Anacardiaceae). Two groups of seedlings 12 months-old were selected, one used as a control and the other to be treated with cement dust. A total of $900 \mathrm{~g}$ of dust was pulverized during five months, twice a week. For both groups of plants periodical visual observations were made to detect possible anomalies. Evaluations of height growth, foliar area, foliar fresh and dry weight, chemical composition of leaves and chlorophyll concentration were also performed. The plants treated with dust presented yellowish leaves, which subsequently became necrotic and felt down. The stems of plants showed disturbance in growth becoming tortuous. The dusted plants also showed significant increase in the concentrations of chlorophylls a, b and total, as well as in macro and micronutrients, $\mathrm{Al}$ and $\mathrm{Na}$, mainly in the leaves not rinsed before analysis. Fresh and dry leaves weights were smaller in the dusted plants than in the control ones. The results indicate this species as useful for bio-monitoring studies because of its sensitivity to cement dust contamination.

Keywords: Brazilian pepper tree; pollution, forest nutrition; active bio-monitoring.
\end{abstract}

\section{INTRODUÇÃO}

Programas de monitoramento ambiental apresentam como principal objetivo a seleção do método mais apropriado para avaliar os níveis de contaminação por substâncias tóxicas em relação aos padrões da qualidade do ar e da água. A utilização desses métodos visa à proteção da saúde humana, preservação da biota e seus hábitats (BURTON, 1986). 
Existem várias técnicas para o monitoramento ambiental, entre as quais o monitoramento biológico (ou biomonitoramento), que pode ser definido como qualquer técnica que se utilize da mensuração de respostas de organismos vivos em relação às alterações ocorridas no seu meio natural (BURTON, 1986). Para a detecção de informações quantitativas e qualitativas referentes à poluição atmosférica, dois métodos podem ser utilizados: o monitoramento passivo e o monitoramento ativo. $\mathrm{O}$ monitoramento passivo, também conhecido como o de campo, utiliza-se das alterações apresentadas por indivíduos (agentes passivos) de uma determinada população ou comunidade, que se encontram naturalmente em uma área sujeita à poluição atmosférica. O ativo ou experimental, por sua vez, utiliza-se de amostragens de plantas (agentes ativos) que são submetidas a dosagens de um ou mais contaminantes, em laboratórios equipados ou na própria área de estudo, procurando-se estabelecer uma correlação entre estes e seus respectivos efeitos (FLORES, citado por MAZZONI-VIVEIROS, 1996; LARCHER, 2000).

Várias espécies - e mesmo as variedades de uma espécie - diferem na sensibilidade e na capacidade de adaptação quando expostas a poluição ou perturbações (GRATANI et al., 2000). Algumas plantas são mais sensíveis aos contaminantes ambientais e, por serem sedentárias, estão sempre expostas às emissões. Por esse motivo, elas podem ser usadas com maior eficiência para a bioindicação de poluentes (LARCHER, 1986).

A poluição por poeira não recebe a mesma atenção dada aos poluentes gasosos, tais como $\mathrm{SO}_{2}$, $\mathrm{NO}_{\mathrm{x}}$ e $\mathrm{O}_{3}$. Porém, estudos detalhados da deposição de poeira sobre a vegetação sugerem que os efeitos podem ser importantes e merecedores de maior atenção (FARMER, 1993).

A poeira de fábricas de cimento pode afetar a vegetação de diversas maneiras. Sua ação sobre o vegetal, para alguns pesquisadores, é quase que exclusivamente mecânica. Isso decorre da composição química da poeira, que forma uma crosta ao se depositar sobre uma superfície e sofrer hidratação, através do orvalho, névoa ou garoa (PEIRCE, 1909; CZAJA, citado por MANDRE; TUULMETS, 1997; SINGH; RAO, 1981). Essa camada solidificada exerce uma modificação no microambiente foliar e várias alterações podem ocorrer no vegetal. Entre elas, são citadas alterações morfológicas da folha, como rachadura, escamação e murcha (SINGH; RAO, 1980; UMA et al., 1994), alterações fisiológicas, como queda na taxa de transpiração estomática e cuticular (SINGH; RAO, 1981; MANDRE; TUULMETS, 1997), alterações anatômicas, como diminuição da densidade estomática (PRASAD; INAMDAR, 1990a; PRASAD; INAMDAR, 1990b; SIBAK; GULYAS, 1990; PANES; ZAMORA, 1991) e alterações enzimáticas, como queda da atividade da enzima catalase (BORKA, 1980; ISMAIL; AHMED, 1984; MIGAHID; ELDARIER, 1995) e da concentração de clorofila (CZAJA, citado por MANDRE; TUULMETS, 1997; LERMAN, 1974; UMA et al., 1994). Redução do processo fotossintético também é relatada na literatura (LAL; AMBASHT, 1982). Como consequência dessa queda, o processo metabólico subsequente (formação de compostos orgânicos ricos em energia, tais como proteínas, gorduras e óleos) também se torna menor. Com isso, uma rede de efeitos é gerada, alterando toda a produção de biomassa do vegetal, o que acarreta baixa produtividade de frutos e sementes, bem como compromete a própria formação dos órgãos reprodutivos e a fertilização do vegetal (SINGH; RAO, 1980; BORKA, 1980; BORKA, 1981; PANDEY et al., 1996; DURGE; PHADNAWIS, 1998; RAO; NARAYANAN, 1998).

Em termos nutricionais, Lal; Ambasht (1982) observaram que as concentrações de Ca, K, Na e P foram aumentados nas folhas de Psidium guayava L. (Myrtaceae) cobertas pela poeira de cimento, quando comparadas com folhas limpas. Os autores comentam que, após o pico máximo de concentração, tanto nas folhas cobertas pela poeira quanto nas limpas, a concentração começou a declinar, mas essa redução foi muito mais lenta nas folhas poluídas. As seguintes justificativas são apresentadas: ocorreu um aumento desses cátions no solo afetado pela poeira de cimento. A incorporação cuticular desses cátions pode ocorrer a partir da crosta depositada na superfície foliar, e a penetração de cátions é facilitada pelo alto $\mathrm{pH}$ nas folhas empoeiradas.

A espécie Schinus terebinthifolius Raddi (Anacardiaceae) é popularmente conhecida como aroeira, aroeira-vermelha, aroeira-mansa, aroeira-branca e árvore-da-pimenta, entre muitos outros nomes (BAGGIO, 1988; LORENZI, 1992). Caracteriza-se por apresentar folhas compostas, alternas, imparipinadas, que têm como detalhe principal pecíolo alado típico. As flores, brancas e melíferas, são de tamanho bastante reduzido, reunidas em cachos terminais. Os frutos globosos são de coloração vermelhovivo a purpúreo ou rosa forte e se encontram agrupados em cachos (REITZ et al., 1983; FLEIG, 1989; LORENZI, 1992). Com grande plasticidade ecológica, a espécie se distribui desde o Nordeste do Brasil, passando pelos cerrados, chegando ao Rio Grande do Sul e estendendo-se até a Argentina e Paraguai 
(BAGGIO, 1988). É comum ser encontrada em beira de rios, córregos e várzeas úmidas de formações secundárias. Contudo cresce em terrenos secos e pobres. É amplamente disseminada por pássaros, o que explica a sua regeneração natural e sua importância na recuperação de áreas degradadas. As árvores podem ser utilizadas para arborização urbana, porém podem causar alergia às pessoas sensíveis (LORENZI, 1992).

A partir da utilização de plantas jovens de Schinus terebinthifolius, este trabalho teve como objetivo avaliar, comparativamente, o desenvolvimento de plantas sujeitas ou não à aplicação de poeira de fábrica de cimento, considerando os seguintes parâmetros: altura das plantas, área foliar, massas fresca e seca das folhas, concentração de clorofila e composição química foliar.

\section{MATERIAL E MÉTODOS}

\section{Condução do experimento}

Indivíduos jovens de Schinus terebinthifolius foram selecionados para um estudo de pulverização com a poeira de cimento, denominado biomonitoramento ativo. Para isso, foram obtidas 60 mudas do viveiro Itrópica, com idade aproximada de um mês. Essas mudas foram replantadas em vasos (capacidade de $6 \mathrm{dm}^{3}$ ) e ficaram em ambiente aberto por aproximadamente onze meses. Então, com doze meses de idade, elas foram separadas em dois grupos de 30 indivíduos com alturas padronizadas, um para controle e outro para ser submetido à poeira de cimento. $\mathrm{O}$ experimento foi conduzido em duas miniestufas feitas de estrutura de madeira e cobertas com plástico transparente, instaladas no Departamento de Solos e Engenharia Agrícola da Universidade Federal do Paraná. Após um mês e quinze dias do início do experimento, uma ventania destruiu as estufas e toda a poeira pulverizada foi lavada da superfície foliar em episódios de chuva. Após um mês, em novembro de 2007, com a construção de novas miniestufas, foi reiniciada a pulverização. O estudo foi conduzido durante cinco meses, sendo o término em abril de 2008. As pulverizações foram feitas duas vezes por semana, e a quantidade total de poeira aplicada foi de aproximadamente $900 \mathrm{~g}$.

Durante o experimento, foram acompanhados o surgimento de sintomas visíveis, como manchas (clorose e necrose), abscisão foliar, rebrota e arquitetura da planta.

Devido ao surgimento de sintomas de desordem nutricional nas plantas pulverizadas, todas as plantas, de ambos os tratamentos, receberam seis doses, a cada dois dias, de $250 \mathrm{ml}$ de solução nutritiva completa de Hoagland (HOAGLAND; ARNON, 1950), quando apresentavam aproximadamente 17 meses de idade, em fevereiro de 2008.

\section{Parâmetros avaliados}

Altura das plantas

Antes de iniciar a pulverização, os dois grupos de indivíduos jovens de S. terebinthifolius foram padronizados em relação à altura, de maneira que os dois grupos apresentassem plantas de mesmo porte. Posteriormente, ao término do ensaio de pulverização, a altura foi novamente avaliada em todas as plantas, com o auxílio de uma régua métrica.

Massas fresca e seca das folhas

Para avaliação das massas fresca e seca das folhas das plantas, foi feita a amostragem de uma folha inteira - folíolos, ráquis e pecíolo - de cada um dos 30 indivíduos de cada estufa. Para tanto, padronizou-se como amostra a quarta folha a partir do ápice da planta. As amostras foram lavadas e, após a remoção do excesso de água, pesadas em balança de precisão. Para a massa seca, as folhas foram prensadas e submetidas à secagem à temperatura de $60^{\circ} \mathrm{C}$, até atingirem peso constante, sendo novamente pesadas.

Área foliar

A área foliar foi obtida a partir das mesmas folhas selecionadas para a análise da massa fresca. A leitura foi feita com auxílio de um scanner e do software WinRIZHO (2003).

Clorofilas a, b e total

A extração de clorofila foi feita num total de 60 amostras de folhas do quarto nó, sendo 30 das plantas controle e 30 das plantas pulverizadas. Para a quantificação dos teores de pigmentos foliares 
(clorofila a, clorofila b e clorofila total), foi efetuada a extração com dimetil sulfóxido (DMSO), com amparo da metodologia desenvolvida por Barnes et al. (1992).

Composição química das folhas

Duas folhas do $4^{\circ}$ ou $5^{\circ}$ nó de cada indivíduo dos dois grupos - controle e pulverizado - foram coletadas e divididas em dois tratamentos - folhas lavadas e não lavadas - e identificadas da seguinte forma: FCL (folhas controle lavadas); FCN (folhas controle não lavadas); FPL (folhas pulverizadas lavadas) e FPN (folhas pulverizadas e não lavadas). Amostras compostas foram formadas a cada três indivíduos, totalizando dez repetições para cada tratamento. Posteriormente, essas folhas foram secadas em estufa a $60{ }^{\circ} \mathrm{C}$, até atingirem peso constante, e, então, moídas.

As metodologias usadas para análise dos elementos químicos estão detalhadamente descritas em Martins; Reissmann (2007). A digestão foi feita em mufla a $500{ }^{\circ} \mathrm{C}$, sendo as cinzas solubilizadas em HCL 3 mol. $\mathrm{L}^{-1}$. Os seguintes elementos foram analisados: cálcio $(\mathrm{Ca})$, magnésio $(\mathrm{Mg})$, sódio $(\mathrm{Na})$, potássio $(\mathrm{K})$, alumínio $(\mathrm{Al})$, cobre $(\mathrm{Cu})$, ferro $(\mathrm{Fe})$, manganês $(\mathrm{Mn})$ e zinco $(\mathrm{Zn})$.

$\mathrm{Na}$ e $\mathrm{K}$ foram lidos por espectrofotometria de emissão em fotômetro de chama em aparelho Digimed NK 2000. Ca, Mg, Al, Cu, Fe, Mn e Zn foram determinados por espectrofotometria de absorção atômica em equipamento Shimadzu, modelo AA-6200.

\section{Análises estatísticas}

$\mathrm{O}$ delineamento utilizado foi o inteiramente casualizado. O teste de Bartlett comprovou a homogeneidade de variâncias, possibilitando a aplicação do teste de Tukey-Kramer HSD a 5\%. Nos casos em que os contrastes eram ortogonais entre si (grau de liberdade dos tratamentos é igual ao grau de liberdade da soma das comparações), utilizou-se o teste t de Student a 5\%, conforme Banzatto; Kronka (1992). Os dados que não apresentaram homogeneidade das variâncias ( $\mathrm{Al}, \mathrm{Fe}$ e $\mathrm{Zn}$ ) foram transformados para escala logarítmica. No caso do parâmetro altura das plantas, os dados não apresentaram homogeneidade das variâncias, mesmo após transformação dos dados, sendo, por isso, utilizado o teste não paramétrico de Wilcoxon/Kruskal-Wallis. As análises foram feitas com o auxílio do software JMP 7.0 (2007).

\section{RESULTADOS E DISCUSSÃO}

\section{Sintomas visíveis}

Durante o desenvolvimento deste estudo, foi possível observar algumas alterações visíveis nas plantas pulverizadas com poeira. Uma dessas alterações foi o surgimento de uma mancha bem visível, observada principalmente no ápice foliolar de folhas maduras (Figura 1). Essa mancha se caracterizou por uma clorose de coloração amarela bastante intensa, que ia se alastrando do ápice para o interior do folíolo. Acompanhando essa mancha, ocorria uma necrose localizada bem no ápice, porém essa necrose não chegava a tomar o folíolo todo, uma vez que a queda do mesmo ocorria antes. Essa alteração não foi observada nos indivíduos do tratamento controle. O aparecimento desses sintomas ocorreu em dois eventos: o primeiro foi após 45-60 dias de pulverização, ocorrendo, em seguida, a abscisão foliar; o segundo evento foi após 90-100 dias, também seguido de abscisão. A queda de folhas não foi avaliada nesse experimento, porém foi possível observar que a abscisão foi intensamente maior nas plantas pulverizadas e vinculada, principalmente, ao surgimento das manchas. $\mathrm{Na}$ literatura disponível, não foram encontrados relatos de situações semelhantes, o que dificulta ainda mais o entendimento e a discussão do processo. Em um trabalho desenvolvido com Myracrodruon urundeuva Allemão (Anacardiaceae), sob o estresse salino, sintomas de toxidez foram relatados. Eles se expressavam na forma de manchas avermelhadas ao longo das nervuras foliares, inicialmente em folhas velhas, evoluindo para um amarelecimento/avermelhamento do limbo foliar, queima das bordas e ápice foliar e queda das folhas em estágio mais avançado (SILVA et al., 2000). Apesar das diferenças no desenvolvimento das manchas, uma possível fonte de toxidez, em função da aplicação da poeira de cimento, não pode ser descartada. Dentre as hipóteses levantadas, têm-se a tentativa de eliminação de alguma substância tóxica presente no interior da folha. Devido à configuração da necrose, acredita-se que ela não tenha ocorrido via floema, pois não aparentava ter conexão com as nervuras, como relatado na toxidez de $M$. urundeuva.

Durante o experimento, foi observado um evento de queda foliar também nas plantas do tratamento controle, porém ele foi bastante discreto, sendo realmente notado com a rebrota de novas folhas. Com isso, acredita-se que tenha sido em função de um evento natural da espécie, pois essa rebrota 
foi notada, também, em indivíduos adultos presentes próximo à área experimental. Nas plantas pulverizadas, a arquitetura da planta foi bastante alterada em função dos episódios de queda foliar. Esse fato não ocorreu nas plantas do tratamento controle. As plantas pulverizadas apresentaram um caule com desenvolvimento mais tortuoso e, em muitos casos, com a copa concentrada em um dos lados da planta. Já os indivíduos controle apresentaram caules mais retilíneos e a copa distribuída de forma mais homogênea. Moraes (1999) também relata diferenças na arquitetura das copas de Tibouchina pulchra (Cham.) Cong. (Melastomataceae) expostas em áreas poluídas de Cubatão, SP, em que elas apresentaram distância reduzida entre os nós e folhas recurvadas, dando à copa um aspecto mais arredondado.

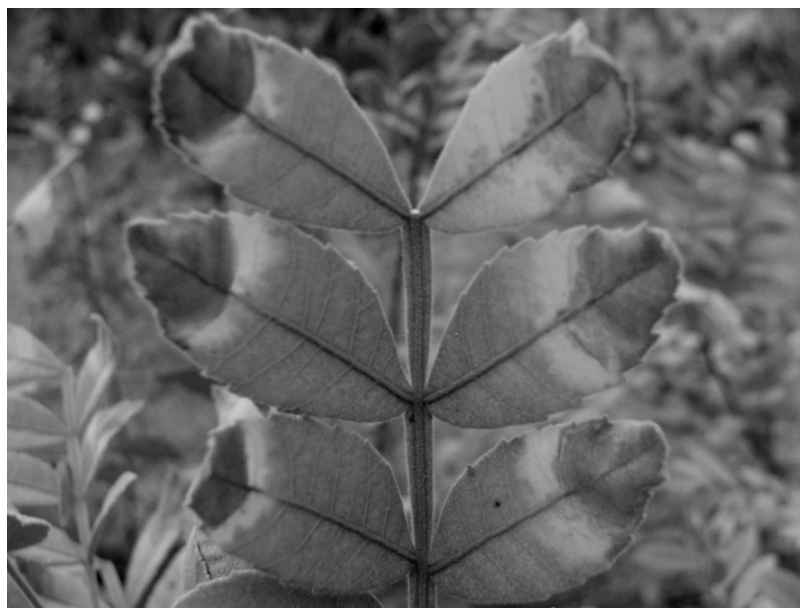

Figura 1. Folha de um indivíduo jovem de Schinus terebinthifolius Raddi (Anacardiaceae), pulverizado com poeira de cimento, com injúrias visíveis na forma de cloroses e necroses apicais.

Figure 1. Leaf of Schinus terebinthifolius Raddi (Anacardiaceae) dusted with cement dust with visible leaf apical injury - yellowish followed by necrosis.

\begin{abstract}
Altura das plantas
As plantas pulverizadas não apresentaram diferenças no crescimento em altura quando comparadas às plantas-controle (Tabela 1).

Muitos pesquisadores têm proposto que poluentes atmosféricos influenciam o crescimento vegetal, principalmente por alterar a alocação de assimilados e matéria seca entre as partes vegetais, como folhas, caules, ramos e raízes (MANDRE; OTS, 1999). Essa alteração pode privilegiar alguns órgãos do vegetal que são essenciais, naquele momento, para a sobrevivência do indivíduo que se encontra sob o estresse. Isso pode revelar que o crescimento em altura, quando a planta se encontra sob o estresse, não é prioritário para a sua sobrevivência. Por isso, esperava-se que os indivíduos pulverizados apresentassem uma redução no crescimento, destinando a energia para a produção e/ou para a manutenção de outros órgãos, como os fotossintetizantes ou reprodutivos. Porém vale ressaltar que os indivíduos-controle também apresentaram problemas nutricionais, evidenciados por folhas cloróticas, sem necroses nessas plantas. Por esse motivo, foram feitas aplicações de solução nutritiva nos dois tratamentos, após um mês e quinze dias do reinício da pulverização, o que pode ter induzido respostas em crescimento semelhantes em ambos os tratamentos.
\end{abstract}

\title{
Área foliar
}

As plantas de ambos os tratamentos não apresentaram diferenças significativas em relação à área foliar ( $p>0,05)$ (Tabela 1). A média foi um pouco mais alta nas plantas-controle - por volta de $4 \%$.

A literatura registra diferenças na área foliar, ocorrendo, geralmente, um aumento desta nos indivíduos sob o impacto da poeira de cimento. Isso ocorre devido ao sombreamento gerado pela deposição, semelhante a uma folha num ambiente sombreado, que tende a apresentar folhas maiores do que as que se encontram em ambientes ensolarados. Esse fato pode ser verificado no trabalho desenvolvido por Anda (1986), em estudo que avaliava o impacto da poeira de cimento em milho. Para 
um experimento, o valor foi $6 \%$ mais expressivo, e para outro, 17,5\% maior do que o controle. Porém o trabalho não entra em detalhes das possíveis causas que acarretaram o aumento no índice da área foliar.

\section{Massas fresca e seca das folhas}

Diferenças significativas foram observadas entre indivíduos-controle e pulverizados em relação às massas fresca e seca. As plantas pulverizadas apresentaram menores médias nos dois parâmetros avaliados, como pode ser conferido na tabela 1. A massa fresca apresentou redução de $13,8 \%$, e a massa seca, de $18 \%$.

Escassas são as menções na literatura referentes às alterações das massas fresca e seca das folhas em resposta à ação da poeira de cimento, porém há citações de uma série de alterações quanto à fitomassa e biomassa de caules, ramos, brotos e raiz. Singh; Rao (1980) observaram queda na quantidade de fitomassa em plantas de trigo e Uma et al. (1994) relataram baixa produção de fitomassa em Crotalaria juncea L.(Fabaceae). Estudos com Pinus sp. revelam que a carga de poeira, a duração do efeito, a composição química e a tolerância da planta, quando se trata da poluição por poeira, podem gerar respostas negativas e positivas no crescimento e formação de biomassa das plantas. Porém a grande maioria descreve mudanças essencialmente negativas na fisiologia e bioquímica das plantas sob altos níveis de impacto alcalino. Essas alterações estão diretamente ligadas a fatores que incluem modificações na absorção de luz pela camada de poeira nas folhas fotossintetizantes, injúrias nos cloroplastos, interferências nas trocas gasosas em consequência da obstrução dos estômatos por partículas de poeira, redução da área fotossintética e mudanças desfavoráveis nas propriedades físico-químicas de solos afetados (vários autores, citados por SINGH; RAO, 1980). No entanto, em áreas onde a carga de poeira é relativamente baixa, o crescimento de acículas, brotos e o incremento radial das árvores de Pinus sp. são estimulados. $\mathrm{O}$ aumento na proporção de biomassa nas acículas de brotos em áreas poluídas sugere que, sob condições desfavoráveis, mecanismos de compensação são iniciados. A planta, assim, aumenta a biomassa com o uso de reservas para sobreviver sob o estresse. Essa hipótese é reforçada pelo fato de que, sob estresse, o número de acículas por broto aumentou em todas as espécies estudadas por Mandre et al. (1999), sendo elas Pseudotsuga menziesii (Mirb.) Franco (Pinaceae), Picea abies (L.) H. Karst. (Pinaceae), Pinus sylvestris L., Picea glauca (Moench) Voss (Pinaceae) e Picea mariana (Mill.) Britton, Sterns e Poggenb. (Pinaceae).

Tabela 1. Valores médios da altura, da área foliar e das massas fresca e seca das plantas de Schinus terebinthifolius Raddi (Anacardiaceae) submetidas ou não à aplicação de poeira alcalina.

Table 1. Average values of height, foliar area and fresh and dry weight of Schinus terebinthifolius Raddi (Anacardiaceae) control plants and pulverized plants.

\begin{tabular}{lcc}
\hline Parâmetro/Local & Controle & Pulverizado \\
\hline Altura* $(\mathrm{m})$ & $1,27^{\mathrm{a}}( \pm 0,02)$ & $1,28^{\mathrm{a}}( \pm 0,05)$ \\
Área foliar $\left(\mathrm{cm}^{2}\right)$ & $50,0^{\mathrm{a}}( \pm 2,41)$ & $48,0^{\mathrm{a}}( \pm 2,84)$ \\
Massa fresca $(\mathrm{g})$ & $1,16^{\mathrm{a}}( \pm 0,06)$ & $0,97^{\mathrm{b}}( \pm 0,06)$ \\
Massa seca $(\mathrm{g})$ & $0,50^{\mathrm{a}}( \pm 0,02)$ & $0,41^{\mathrm{b}}( \pm 0,02)$ \\
\hline
\end{tabular}

*Teste não-paramétrico de Kruskal-Wallis. Valores seguidos pela mesma letra não diferem entre si pelo teste t de Student's $(\mathrm{p}>0,05) ;()=$ erro padrão

\section{Concentração de clorofila}

Foi observado um aumento significativo nas concentrações de clorofila a, b e total nos indivíduos que se encontravam sob o impacto da poeira de cimento $(\mathrm{p}<0,05)$ (Tabela 2).

Os seguintes aumentos foram observados: $14,53 \%$ para clorofila a, $18,75 \%$ para clorofila b e $23,2 \%$ para clorofila total. A relação clorofila $\mathrm{a} / \mathrm{b}$ foi $23,5 \%$ maior nas plantas que foram expostas à poeira.

O nível de irradiação é um importante fator ecológico do qual todas as plantas autotróficas dependem. Baixa intensidade luminosa causa estresse para as plantas porque a irradiação limita a fotossíntese e, assim, o "ganho" de carbono e o crescimento vegetal (LAMBERS et al., 1998). Acreditase que, devido à formação da camada de poeira sobre a superfície foliar, ocorreu um sombreamento artificial. De acordo com Larcher (2000), a concentração de clorofila em folhas sombreadas é maior do que em folhas de sol, enquanto que a relação clorofila a/b é reduzida. Segundo Mandre; Tuulmets (1997), foi observada diminuição na proporção de clorofila a/b em acículas de Picea abies de 1 ano de idade sob o impacto da poeira de cimento, numa distância de $0,5 \mathrm{~km}$ da fábrica, com uma diferença de $25 \%$ comparada ao controle. Porém, em distâncias maiores que $1,5 \mathrm{~km}$, a diferença na proporção de clorofila 
a/b não foi significativamente diferente. Neste estudo, as plantas de $S$. terebinthifolius pulverizadas apresentaram maior proporção de clorofila a/b, resultado contrário ao relatado na literatura.

Tabela 2. Valores médios da concentração de clorofila ( $\mathrm{mmol} / \mathrm{mg}$ ) das plantas de Schinus terebinthifolius Raddi (Anacardiaceae) submetidas ou não à aplicação de poeira alcalina.

Table 2. Average values of chlorophyll $(\mathrm{mmol} / \mathrm{mg}$ ) in plants of Schinus terebinthifolius Raddi (Anacardiaceae) control plants and pulverized plants.

\begin{tabular}{lcc}
\hline Parâmetro/Local & Controle & Pulverizado \\
\hline Clorofila a & $1,90^{\mathrm{a}}( \pm 0,06)$ & $2,34^{\mathrm{b}}( \pm 0,08)$ \\
Clorofila b & $0,65^{\mathrm{a}}( \pm 0,02)$ & $0,80^{\mathrm{b}}( \pm 0,03)$ \\
Clorofila total & $2,55^{\mathrm{a}}( \pm 0,08)$ & $3,13^{\mathrm{b}}( \pm 0,10)$ \\
Proporção de clorofila a/b & $3,00^{\mathrm{a}}( \pm 0,12)$ & $3,10^{\mathrm{a}}( \pm 0,15)$ \\
\hline
\end{tabular}

Valores seguidos pela mesma letra não diferem entre si pelo teste t de Student's $(p>0,05)$. ( ) = erro padrão.

Shukla et al. (1990) observaram um fraco aumento na concentração de clorofila em Brassica campestris L. (Brasicaceae), que receberam uma carga de $3 \mathrm{~g}$ de poeira diariamente durante 30 dias. Esse incremento deve ser atribuído ao efeito de sombreamento da folha que recebeu a poeira de cimento. Porém, posteriormente, ocorreu uma queda da concentração da clorofila, devido ao aumento da carga de poeira pulverizada e ao tempo maior de exposição. Saarinen (1993, apud Gratani et al., 2000) e Carreras et al. (1996) observaram aumento na concentração de clorofila em árvores adultas de Ligustrum lucidum W. T. Aiton (Oleaceae) e de Quercus ilex L. (Fagaceae), sob o impacto da poluição urbana, e relataram que esse aumento pode ser considerado um mecanismo de resistência ao nível foliar em árvores que se encontram em áreas poluídas.

Dessa forma, acredita-se que o aumento da concentração de clorofila nos indivíduos empoeirados de Schinus terebinthifolius esteja relacionado ao efeito de sombreamento gerado pela deposição da poeira, mas também pode estar vinculado aos eventos de abscisão foliar, e essa resposta seria um mecanismo de resistência para melhorar o seu desempenho frente ao estresse imposto pela poeira. Segundo Larcher (2000), as plantas apresentam capacidade de adaptação para a composição espectral da luz. Foi primeiramente demonstrado ocorrer em algas azuis e vermelhas, as quais mudam as quantidades de seus pigmentos em função da qualidade espectral da luz que chega até a profundidade onde se encontram. Em plantas terrestres, essa adaptação também foi observada, ocorrendo alteração na composição dos pigmentos nos cloroplastos. A capacidade da planta em compensar as mudanças na radiação utilizando seus pigmentos é uma importante vantagem ecológica. Como a queda dos folíolos foi bastante intensa, ocorreu uma redução maior da área fotossintética, que, associada a uma menor taxa de luz incidente, talvez tenha estimulado a planta a compensar a pequena área fotossintética com um aumento da concentração de clorofila, para melhorar o seu desempenho de produção energética.

\section{Composição química foliar}

Nas tabelas 3 e 4 são apresentados, respectivamente, os resultados referentes às concentrações médias foliares dos macronutrientes $\mathrm{Ca}, \mathrm{Mg}$ e $\mathrm{K}$, e dos micronutrientes $\mathrm{Cu}, \mathrm{Fe}, \mathrm{Mn}$ e $\mathrm{Zn}$, além das concentrações de Na e Al. Pode-se observar que, para quase todos os elementos, as folhas provenientes dos indivíduos pulverizados e que não foram lavadas foram as que apresentaram as maiores médias, tanto para os macros quanto para os micronutrientes. Esse resultado já era esperado, uma vez que esses elementos estão presentes na composição química da poeira de cimento, como descrito no trabalho de Mandre; Tuulmets (1997). As exceções ficaram para K e Zn. No caso do potássio, a maior média foi encontrada nas folhas pulverizadas, porém lavadas. Apesar desse elemento ser altamente susceptível ao processo de lixiviação, a absorção via foliar pode ocorrer (ARCOVA; CICCO, 1987; FREITAS et al., 2007), o que justificaria essa maior média nas folhas pulverizadas mesmo lavadas. Já o zinco apresentou a maior média nas folhas-controle não lavadas, porém, comparando as médias entre os tratamentos, é possível observar que elas são bem próximas (Tabela 4), apresentando distinção $(\mathrm{p}<0,05)$ apenas entre as folhas pulverizadas lavadas e as pulverizadas não lavadas.

Muitos autores apontam os problemas de interpretação do estado nutricional usando material não lavado (DRECHSEL; ZECH, 1991; MIYAZAWA et al., 1999) e que se encontram próximos a fontes 
poluidoras (KRIVAN et al., 1987), pois as deficiências nutricionais podem ser mascaradas, e resultados falsos indicando altas concentrações, particularmente de elementos como $\mathrm{Mg}, \mathrm{K}$ e Ca, podem ser gerados. No trabalho desenvolvido por Trimbacher; Weiss (1999), as mais altas concentrações de elementos foram descobertas nas folhas situadas próximas às fontes de emissão, incluindo os macronutrientes $\mathrm{N}, \mathrm{Ca}, \mathrm{K} \mathrm{e}$ $\mathrm{Mg}$, em folhas não lavadas. Com exceção do $\mathrm{K}$, os mesmos resultados foram encontrados neste trabalho.

Tabela 3. Concentração de macronutrientes e $\mathrm{Na}(\mathrm{g} / \mathrm{kg})$ nas folhas de plantas de Schinus terebinthifolius Raddi (Anacardiaceae).

Table 3. Concentration of macronutrients and $\mathrm{Na}(\mathrm{g} / \mathrm{kg})$ in leaves of Schinus terebinthifolius Raddi (Anacardiaceae) plants.

\begin{tabular}{lcccc}
\hline \multirow{2}{*}{ Elemento } & \multicolumn{4}{c}{ Tratamento } \\
\cline { 2 - 5 } & FPN & FPL & FCL & FCN \\
\hline $\mathrm{Ca}$ & $36,1^{\mathrm{a}}( \pm 0,7)$ & $24,0^{\mathrm{b}}( \pm 0,4)$ & $\left.12,0^{\mathrm{c}} \pm 0,2\right)$ & $10,5^{\mathrm{d}}( \pm 0,4)$ \\
$\mathrm{Mg}$ & $6,1^{\mathrm{a}}( \pm 0,2)$ & $5,1^{\mathrm{b}}( \pm 0,1)$ & $5,0^{\mathrm{b}}( \pm 0,06)$ & $4,1^{\mathrm{c}}( \pm 0,1)$ \\
\hline \multirow{2}{*}{$\mathrm{Na}$} & $\mathrm{FPN}$ & FCN & FPL & FCL \\
& $0,8^{\mathrm{a}}( \pm 0,03)$ & $0,7^{\mathrm{b}}( \pm 0,02)$ & $0,6^{\mathrm{b}}( \pm 0,02)$ & $0,6^{\mathrm{b}}( \pm 0,01)$ \\
\hline \multirow{2}{*}{$\mathrm{K}$} & $\mathrm{FPL}$ & FPN & FCN & FCL \\
& $8,1^{\mathrm{a}}( \pm 0,1)$ & $7,6^{\mathrm{b}}( \pm 0,06)$ & $\left.6,0^{\mathrm{c}} \pm 0,1\right)$ & $\left.6,0^{\mathrm{c}} \pm 0,1\right)$ \\
\hline
\end{tabular}

Valores seguidos pela mesma letra não diferem entre si pelo teste de Tukey-Kramer HSD (p>0,05); ( ): erro padrão; FPN: folhas pulverizadas não lavadas; FPL: folhas pulverizadas lavadas; FCN: folhas controle não lavadas; FCL: folhas controle lavadas.

Tabela 4. Concentração dos micronutrientes e do $\mathrm{Al}(\mathrm{mg} / \mathrm{kg})$ nas folhas de plantas de Schinus terebinthifolius Raddi (Anacardiaceae).

Table 4. Concentration of micronutrients and $\mathrm{Al}(\mathrm{g} / \mathrm{kg})$ in leaves of Schinus terebinthifolius Raddi (Anacardiaceae) plants.

\begin{tabular}{lcccc}
\hline \multirow{2}{*}{ Elemento } & \multicolumn{5}{c}{ Tratamento } \\
\cline { 2 - 5 } & FPN & FPL & FCN & FCL \\
\hline $\mathrm{Al}$ & $713,6^{\mathrm{a}}( \pm 126)$ & $463,5^{\mathrm{a}}( \pm 40)$ & $199,5^{\mathrm{b}}( \pm 31)$ & $193,5^{\mathrm{b}}( \pm 37)$ \\
$\mathrm{Cu}$ & $8,1^{\mathrm{a}}( \pm 0,3)$ & $6,4^{\mathrm{b}}( \pm 0,3)$ & $5,4^{\mathrm{b}}( \pm 0,2)$ & $5,3^{\mathrm{b}}( \pm 0,3)$ \\
$\mathrm{Fe}$ & $624,1^{\mathrm{a}}( \pm 26)$ & $437,7^{\mathrm{b}}( \pm 10,3)$ & $170,2^{\mathrm{c}}( \pm 5,5)$ & $126,1^{\mathrm{d}}( \pm 5,6)$ \\
\hline \multirow{2}{*}{$\mathrm{Mn}$} & $\mathrm{FPN}$ & FCL & FPL & FCN \\
& $50,1^{\mathrm{a}}( \pm 1,3)$ & $40,4^{\mathrm{b}}( \pm 1,1)$ & $40,1^{\mathrm{b}}( \pm 2,0)$ & $39,7^{\mathrm{b}}( \pm 1,0)$ \\
\hline \multirow{2}{*}{$\mathrm{Zn}$} & $\mathrm{FCN}$ & FPN & FCL & FPL \\
& $12,0^{\mathrm{a}}( \pm 0,5)$ & $11,8^{\mathrm{a}}( \pm 0,3)$ & $11,2^{\mathrm{a}}( \pm 0,4)$ & $10,0^{\mathrm{b}}( \pm 0,2)$ \\
\hline
\end{tabular}

Valores seguidos pela mesma letra não diferem entre si pelo teste de Tukey-Kramer HSD (p>0,05); ( ): erro padrão; FPN: folhas pulverizadas não lavadas; FPL: folhas pulverizadas lavadas; FCN: folhas controle não lavadas; FCL: folhas controle lavadas.

O maior incremento observado, dentre os macronutrientes, foi o do cálcio, que apresentou um aumento de $71 \%$ em relação ao controle não lavado e de $50 \%$ em relação ao controle lavado, ambos apresentando diferenças significativas $(\mathrm{p}<0,05)$. Em segundo lugar encontra-se o magnésio, que apresentou um aumento de 32,8\% nas folhas pulverizadas não lavadas, quando comparadas às folhas controle não lavadas $(\mathrm{p}<0,05)$. Aqui, o interessante é notar a diferença entre as folhas controle lavadas e pulverizadas lavadas - apenas $2 \%$-, indicando a riqueza do elemento na poeira de cimento. Fato semelhante pode ser observado para o sódio, que apresentou um aumento de $12,5 \%(\mathrm{p}<0,05)$ nas folhas pulverizadas não lavadas e apenas $6 \%(p>0,05)$ de diferença entre as folhas controle e pulverizadas lavadas.

Como citado anteriormente, no caso do potássio, o maior incremento foi nas folhas pulverizadas lavadas (26\%), enquanto que as folhas pulverizadas não lavadas apresentaram um aumento de $21 \%$. Em ambos os tratamentos as diferenças foram estatisticamente significativas $(\mathrm{p}<0,05)$.

Entre os micronutrientes, o maior incremento, comparado ao respectivo controle, foi o do ferro: $73 \%$ nas folhas pulverizadas não lavadas. Nas folhas pulverizadas lavadas, a diferença foi de $71 \%$, sendo ambas estatisticamente significativas $(\mathrm{p}<0,05)$. Isso demonstra que o ferro não foi eliminado durante a 
lavagem das folhas e que, provavelmente, estava incorporado à folha. Como a diferença é bastante grande em relação ao controle - inclusive nas folhas lavadas -, sugere-se que as plantas pulverizadas absorveram esse ferro excedente da poeira de cimento. Outro elemento que apresentou uma grande concentração nas folhas pulverizadas foi o alumínio, $72 \%$ maior nas folhas pulverizadas não lavadas e 58,2\% maior nas folhas pulverizadas lavadas, evidenciando que ocorreu o mesmo que o ferro, pois, apesar da lavagem, a concentração ainda foi bem mais alta, indicando que o alumínio pode ter sido adquirido da poeira de cimento.

Cobre e manganês apresentaram como resultado maior concentração nas folhas das plantas pulverizadas e não lavadas, $33,3 \%$ e $24 \%$, respectivamente $(\mathrm{p}<0,05)$. No caso do cobre, a porcentagem ainda foi razoavelmente alta nas folhas lavadas das plantas pulverizadas (17\%), porém essa diferença não foi estatisticamente significativa ( $p>0,05)$.

\section{CONCLUSÕES}

Com base nos resultados obtidos, conclui-se que a poeira de cimento atuou como um fator estressante para os indivíduos contaminados. Entretanto, a espécie utilizada apresenta diferentes mecanismos de reação a esse impacto, como é o caso do aumento da concentração de clorofila. Por ser sensível à contaminação, mas ao mesmo tempo resistente, essa espécie caracteriza-se como adequada para os estudos de biomonitoramento em áreas sujeitas à poluição por poeira de cimento.

\section{REFERÊNCIAS}

ANDA, A. Effect of cement kiln dust on the radiation balance and yields of plants. Environmental Pollution. Series A. Ecological and biological, London, v. 40, n. 3, p. 249-256, 1986.

ARCCOVA, F. C. S.; CICCO, V. Fluxo de nutrientes através da precipitação interna e escoamento pelo tronco em floresta natural secundária no Parque Estadual da Serra do Mar - Núcleo Cunha - SP. Boletim Técnico do Instituto Florestal, São Paulo, v. 41, n. 1, p. 37-58, 1987.

BAGGIO, A. J. Aroeira como potencial para usos múltiplos na propriedade rural. Boletim de Pesquisa Florestal, Curitiba, v. 17, p. 25-32, 1988.

BANZATTO, D. A.; KRONKA, S. N. Experimentação agrícola. 2. ed. Jaboticabal: FUNEP, 1992. 247 p.

BARNES, J. D.; BALANGER, L.; MANRIQUE, E.; ELVIRA, S.; DAVISON, A.W. A reappraisal of use of DMSO for extraction and determination of chlorophylls $a$ and $b$ in lichens and higher plants. Environmental and Experimental Botany, Elmsford, v. 32, p. 85-100, 1992.

BORKA, G. The effect of cement dust pollution on growth and metabolism of Helianthus annuus. Environmental Pollution. Series A. Ecological and biological, London, v. 22, p. 75-79, 1980.

BORKA, G. Effect of cement-kiln dust on the maize plant. Acta Agronomica Academiae Scientiarum Hungaricae, Budapest, v. 30, n. 3-4, p. 289-295, 1981.

BURTON, M. A. S. Biological monitoring. London: King's College London: University of London, 1986.

CARRERAS, H. A.; CAÑAS, M. S.; PIGNATA, M. L.. Differences in responses to urban air pollutants by Lugustrum lucidum Ait. and Ligustrum lucidum Ait. f. tricolor (Rehd.) Rehd. Environmental Pollution, Barking, v. 93, n. 2, p. 211-218, 1996.

DRECHSEL, P.; ZECCH, W. Foliar nutrient levels of broad-leaved tropical trees: A tabular review. Plant and soil, The Hague, v. 131, p. 29-46, 1991.

DURGE, D. V.; PHADNAWIS, B. N. Effect of cement dust pollution on yield and attributes of wheat (Triticum aestivum L.). Annals of Plant Physiology, v. 12, n. 1, p. 11-14, 1998.

FARMER, A. M. The effects of dust on vegetation - a review. Environmental Pollution, Barking, v. 79, p. 63$75,1993$. 
FLEIG, M. Anacardiaceas. Flora Ilustrada Catarinense. 1989. 62 p.

FREITAS, R.; LEANDRO, W.; CARVALHO, M. Efeito da adubação potássica via solo e foliar sobre a produção e a qualidade da fibra em algodoeiro (Gossypium hirsutum L.). Pesquisa Agropecuária Tropical, Goiania, v .37, n .2, p. 106-112, 2007.

GRATANI, L.; CRESCENTE, M. F.; PETRUZZI, M. Relationship between leaf life-span and photosynthetic activity of Quercus ilex in polluted urban areas (Rome). Environmental Pollution, Barking, v. 110, p. 19-28, 2000.

HOAGLAND, D.; ARNON, D.I. The water culture method for growing plants without soil. California Agriculture Experimental Station Circular, v. 347, p. 1- 32, 1950.

ISMAIL, F.; AHMED, S. Effect of phytotoxic air pollution on the amino acid content of plants growing in Karachi area. Pakistan Journal of Botany, v.16, n. 2, p. 117-122, 1984.

JMP. JMP 7.0. SAS Institute Inc., 2007.

KRIVAN, V.; SCHALDACH, G.; HAUSBECK, R. Interpretation of Element Analyses of Spruce-needle Tissue Falsified by Atmospheric Surface Deposition. Naturwissenschaften, Berlin, v. 74, p. 242-245, 1987.

LAL, B.; AMBASHT, R. S. Impact of cement dust on the mineral and energy concentration of Psidium guayava. Environmental Pollution. Series A. Ecological and biological, London, v. 29, p. 241-247, 1982.

LAMBERS, H.; III, F. S. C.; PONS, T. L. Plant Physiological Ecology. New York: Springer-Verlag, 1998.

LARCHER, W. Ecofisiologia Vegetal. São Paulo: Pedagógica e Universitária, 1986.

LARCHER, W. Ecofisiologia Vegetal. São Carlos: Rima Artes e Textos, 2000.

LERMAN, S. Cement kiln dust and bean plant (Phaseolus vulgaris L. Black Valentine var.); in-deth invetigations into plant morphology, physiology and pathology. Dissertation Abstracts International, v. 34, n. 10, p. 4775, 1974.

LORENZI, H. Árvores Brasileiras: Manual de Identificação e Cultivo de Plantas Arbóreas Nativas do Brasil. Nova Odessa: Plantarum. 1992.

MANDRE, M.; KLÕSEIKO, J.; OTS, K.; TUULMETS, L. Changes in phytomass and nutrient partitioning in young conifers in the extreme alkaline growth conditions. Environmental Pollution, Barking, v. 105, n. 2, p. 209-220, 1999.

MANDRE, M.; OTS, K. Growth and biomass partitioning of 6-year-old spruces under alkaline dust impact. Water, Air, and Soil Pollution, v. 114, p. 13-25, 1999.

MANDRE, M.; TUULMETS, L. Pigment changes in norway spruce induced by dust pollution. Water, Air, and Soil Pollution, v. 94, p. 247-258, 1997.

MARTINS, A. P. L.; REISSMANN, C. B. Material vegetal e as rotinas laboratoriais nos procedimentos químico-analíticos. Scientia Agraria, Curitiba, v. 8, n. 1, p. 1-17, 2007.

MAZZONI-VIVEIROS, S. C. Aspectos Estruturais de Tibouchina pulchra Cogn. (Melastomataceae) Sob o Impacto de Poluentes Atmosféricos Provenientes do Complexo Industrial de Cubatão, SP-Brasil. Tese (Doutorado em Botânica) - Instituto de Biociências, Universidade de São Paulo, São Paulo, 1996.

MIGAHID, M. M.; EL-DARIER, S. M. Effect of cement dust on three halophytic species of the Mediterranean salt marshes in Egypt. Journal of Arid Environments, London, v. 30, n. 3, p. 361-366, 1995.

MORAES, R. M. Fotossíntese líquida e respostas bioindicadoras da poluição aérea em indivíduos jovens de Tibouchina pulchra Cogn. (Melastomataceae), em Cubatão, SP. Tese (Doutorado em Ecologia) - Instituto de Biociências, Universidade de São Paulo, São Paulo, 1999. 
MYAZAWA, M.; PAVAN, M. A.; MURAOKA, T.; CARMO, C. A. F.; MELlO, W. J. Análises químicas de solos, plantas e fertilizantes. Brasília, DF: EMBRAPA, 1999. p. 171-224.

PANDEY, D. D.; SANJEEV, K.; KUMAR, S. Impact of cement dust pollution on biomass, chlorophyll, nutrients and grain characteristics of wheat. Environmental and Ecology, Jaipur, v. 14, n. 4, p. 872-875, 1996.

PANES, V. A.; ZAMORA, P. M. Leaf epidermical features of four Philippine plants as indicators of cement dust pollution. Philippine Journal of Science, Manila, v. 120, n. 3, p. 249-267, 1991.

PRASAD, M. S. V.; INAMDAR, J.A. Effect of cement kiln dust pollution on black gram (Vigna mungo (L.) Hepper). Proceedings of the Indian Academy of Sciences. Plant Sciences, Bangalore, v. 100, n. 6, p. 435443, 1990a.

PRASAD, M. S. V.; INAMDAR, J. A Effect of particulate pollution on Pisum sativum L. Journal of Phytological Research, v. 3, n. 1-2, p. 71-77, 1990b.

RAO, C. M.; NARAYANAN, A. Growth of rice plants exposed to cement dust pollution. Journal of Research ANGRAU, v. 26, n. 3-4, p. 11-14, 1998.

REITZ, R.; KLEIN, R.M.; REIS, A. Projeto madeira do Rio Grande do Sul. Sellowia, Itajaí, v. 34-35, p. 1-525, 1983.

SHUKLA, J.; PANDEY, V.; SINGH, S. N.; YUNUS, M.; SINGH, N.; AHMAD, K.J. Effect of cement dust on the growth and yield of Brassica campestris L. Environmental Pollution, Barking, v. 66, p. 81$88,1990$.

SIBAK, S.; GULYAS, S. Leaf anatomical changes in perishing acaluous oaks. Acta Universitatis Szegediensis Acta Biologica, v. 36, n. 1-4, p. 43-52, 1990.

SILVA, F. A.; MELLONI, R.; MIRANDA, J. R.; CARVALHO, J. G. Efeito do estresse salino sobre a nutrição mineral e o crescimento de mudas de aroeira (Myracrodruon urundeuva) cultivadas em solução nutritiva. CERNE, Lavras, v. 6, n. 1, p. 52-59, 2000.

SINGH, S. N.; RAO, D. N. Growth of wheat plants exposed to cement dust pollution. Water, Air, and Soil Pollution, v. 14, p. 241-249, 1980.

SINGH, S. N.; RAO, D. N. Certain response of wheat plants to cement dust pollution. Environmental Pollution. Series A. Ecological and biological, London, v. 24, p. 75-81, 1981.

TRIMBACHER, C.; WEISS, P. Needle surface characteristics and element contents of Norway spruce in relation to the distance of emission sources. Environmental Pollution, Barking, v. 105, p. 111-119, 1999.

UMA, C. H.; RAO, T. V. R.; INAMDAR, J. A. Impact of cement kiln dust pollution on sunhemp (Crotalaria juncea L.). Phytomorphology, New Delhi, v. 44, n. 3-4, p. 223-229, 1994.

WinRIZHO. WinRIZHO Pro V2003b. Regent Instruments Inc., 2003. 
